\title{
MicroARN circulantes en sangre de pacientes con cáncer de próstata
}

\author{
Circulating MicroRNAs in blood of patients with prostate cancer
}

\author{
V. Medina-Villaamil ${ }^{\mathrm{a}}$, S. Martínez-Breijo ${ }^{\mathrm{b}}$, P. Portela-Pereira ${ }^{\mathrm{b}}$, M. Quindós- \\ Varela $^{c}$, I. Santamarina-Caínzos ${ }^{\mathrm{a}}$, L.M. Antón-Aparicio ${ }^{\mathrm{c}}$, d, F. Gómez-Veiga ${ }^{\mathrm{b}}$ \\ ${ }^{a}$ Instituto de Investigación Biomédica, A Coruña, INIBIC, CHU A Coruña-XXIAC, A Coruña, España \\ ${ }^{b}$ Servicio de Urología Médica, CHU A Coruña-XXIAC, A Coruña, España \\ ${ }^{c}$ Servicio de Oncología Médica, CHU A Coruña-XXIAC, A Coruña, España \\ ${ }^{d}$ Departamento de Medicina, Universidade da Coruña, UDC, A Coruña, España
}

\begin{abstract}
Resumen
Introducción. Los microARN (miARN) son ARN reguladores de pequeño tamaño que no codifican para proteínas. La detección de células tumorales circulantes (CTC) proporcionaría información diagnóstica y pronóstica en los tumores de próstata (TP). En este sentido los miARN podrían constituir una nueva y prometedora clase de biomarcadores para la detección de CTC.

Objetivos. Analizar miARN circulantes en sangre total como marcadores no invasivos en pacientes con cáncer de próstata localizado e individuos sanos.

Material y métodos. Estudio preliminar con una $\mathrm{N}$ poblacional de 40 pacientes con una media de edad de 71 años y un PSA medio de $18,9 \mathrm{ng} / \mathrm{ml}$ (rango). Respecto al grupo de riesgo (GR): un 33,3\% bajo riesgo, un $30 \%$ riesgo intermedio y un $36,7 \%$ alto riesgo. Se realizó un estudio previo in silico que identificó 92 miARN candidatos seguido de otro in vivo para verificar los hallazgos del primero mediante tecnología de arrays de PCR a tiempo real.

Resultados. El análisis estadístico de los resultados reveló 10 miARN candidatos con una expresión diferencial estadísticamente significativa entre los distintos grupos de riesgo y los controles sanos: hsa-miR337-3p, hsa-miR-330-3p, hsa-miR-339-3p, hsa-miR-124, hsa-miR-218, hsa-miR-128, hsa-miR-10a, hsa-miR199b-5p, hsa-miR-200b y hsa-miR-15b

Conclusiones. Nuestros datos sugieren que los miARN circulantes pueden servir como biomarcadores para identificar grupos de riesgo en $\mathrm{CaP}$.
\end{abstract}

\footnotetext{
Abstract

Introduction. MicroRNAs (miRNAs) are small regulatory RNAs that do not code for proteins. Detection of circulating tumor cells (CTC) would provide diagnostic and prognostic information in prostate tumors (PT). Thus, miRNAs could constitute a promising new class of biomarkers for CTC detection.

Objectives. To analyze circulating microRNAs in whole blood as non-invasive markers in patients with localized prostate cancer and healthy individuals.

Material and methods. A preliminary study including a population of 40 patients with mean age of 71 years and mean PSA of 18, 9ng/ml (range). Regarding the risk group (RG): 33.3\% had low risk, $30 \%$ intermediate risk and $36.7 \%$ high risk. A previous in silico study identified 92 candidates and was followed by another in vivo to verify the findings of the former using array technology by real-time PCR.

Results. Statistical analysis of the results revealed 10 microRNAs candidates with statistically significant differential expression between the different risk groups and healthy controls: hsa-miR-337-3p, hsa-miR-3303p, hsa-miR-339-3p, hsa-miR-124, hsa-miR-218, hsa-miR-128, hsa-miR-10a, hsa-miR-199b-5p, hsa-miR$200 \mathrm{~b}$ and hsa-miR-15b.

Conclusions. Our data suggest that circulating microRNAs can act as biomarkers to identify risk groups in $\mathrm{CaP}$.
} 


\section{Palabras clave}

Cáncer de próstata; MicroARN; Micrometástasis; Reacción en cadena de la polimerasa

Keywords

Prostate cancer; MicroRNAs; Micrometastasis; Polymerase chain reaction

\section{Introducción}

El cáncer de próstata $(\mathrm{CaP})$ es la neoplasia maligna más frecuente de los hombres en los países occidentales y es la segunda causa más común de muerte por cáncer. La exploración rectal (TR) y el antígeno específico de la próstata (PSA) se aplican habitualmente para la detección temprana de CaP. Sin embargo, el valor diagnóstico de ambos métodos es limitado por la baja sensibilidad (TR) o baja especificidad (prueba PSA). Por lo tanto, se hace necesario la búsqueda de nuevos biomarcadores mejorados para el diagnóstico de $\mathrm{CaP}$.

Los microARN (miARN) se ha estimado que modulan la expresión de aproximadamente el $30 \%$ de genes codificadores de proteínas en los humanos ${ }^{1}$. La expresión alterada o disfunción de las vías de miARN puede afectar a procesos celulares divergentes, incluyendo ciclo celular, diferenciación y proliferación, influyendo así en la tumorogénesis y progresión.

Los miARN pueden ser exportados por las células y circulan en la sangre humana en una forma estable, algunos de los miARN circulantes pueden distinguir a los pacientes de los individuos sanos ${ }^{2}$. Estos hallazgos implican su posible uso como marcadores no invasivos para monitorizar la progresión de la enfermedad ${ }^{3}$ and 4 . En el cáncer de próstata varios trabajos previos han demostrado que los miARN derivados de tumores de origen epitelial se pueden detectar en muestras de sangre y algunos se correlacionan potencialmente con el riesgo de progresión de la enfermedad o resultaron predictores de la agresividad de la enfermedad ${ }^{4,5,6}$ and 7 . Así en este trabajo se explora la posibilidad de identificar genes miARN como firmas de material genético circulante en $\mathrm{CaP}$.

\section{Material y métodos}

\section{Muestras clínicas de sangre}

En el periodo comprendido entre septiembre de 2010 y octubre de 201278 pacientes diagnosticados de $\mathrm{CaP}$ no metastásico que no habían recibido tratamiento neoadyuvante hormonal o radioterápico fueron seleccionados para nuestro estudio. Fueron diagnosticados mediante biopsia transrectal ecodirigida con toma de 10 cilindros y previa realización de PSA. De dicha población 30 pacientes fueron elegidos para el análisis inicial, los otros 10 casos del estudio están representados por la población sana $(\mathrm{N}=10)$. La edad media es de 71 años (rango 56-83), PSA total medio de $25,73 \mathrm{ng} / \mathrm{ml}$ (rango 2,2-425), PSA libre 1,09 ng/ml y media de PSA libre/total $17,46 \%$, con una media de $41 \%$ de cilindros positivos y una mediana de 7 para el grado de Gleason. En el $20 \%$ de las muestras está presente la afectación perineural en el estudio de anatomía patológica.

Clasificamos por subgrupos dichos pacientes obteniendo $10(33,3 \%), 9(30,0 \%)$ y $11(36,7 \%)$ pacientes con $\mathrm{CaP}$ de bajo, intermedio y alto riesgo respectivamente. Diez pacientes con cáncer de próstata de bajo riesgo presentan una edad media de 69 años, PSA total medio de 5,82 ng/ml, y media PSA 1/t 19\% con una media del 17\% de cilindros positivos en la biopsia, con Gleason 6 y sin afectación perineural en ninguna muestra. 
Nueve pacientes clasificados como grupo de riesgo intermedio con una edad media de 71 años, PSA total medio de $4,31 \mathrm{ng} / \mathrm{ml}$, y media de PSA $1 / \mathrm{t} 14 \%$. La media de cilindros positivos es del $31 \%$ con una mediana de 7 para el grado de Gleason.

Once pacientes conforman el grupo de alto riesgo con una edad media de 74 años, PSA total medio de $61,36 \mathrm{ng} / \mathrm{ml}$, PSA $1 / \mathrm{t}$ medio $21 \%$, media $77 \%$ de cilindros positivos y mediana de 8 para la escala de Gleason. En el $45 \%$ de los casos observamos datos de infiltración perineural.

Se reclutaron además 10 controles emparejados por media de edad con los individuos afectados de $\mathrm{CaP}$.

Todos los pacientes dieron su consentimiento para donar material biológico, de acuerdo con los requisitos del Comité Ético de Investigación Clínica de Galicia.

Las características clínicas de los pacientes son recogidas en la tabla 1.

Tabla 1. Características clínicas de los pacientes

\begin{tabular}{|c|c|c|}
\hline Características & Cáncer de próstata & Controles sanos \\
\hline \multicolumn{3}{|l|}{ Edad (años) } \\
\hline Media \pm DT & $71 \pm 6$ & $65 \pm 7,5$ \\
\hline Mediana (rango) & $70(56-85)$ & $69(53-74)$ \\
\hline \multicolumn{3}{|l|}{ Estadio clínico } \\
\hline $\mathrm{cT} 1 \mathrm{c}$ & $18(60 \%)$ & \\
\hline cT2a & $10(33,3 \%)$ & \\
\hline $\mathrm{cT} 2 \mathrm{c}$ & $1(3,3 \%)$ & \\
\hline cT3 & $1(3,3 \%)$ & \\
\hline \multicolumn{3}{|l|}{ Grupo PSA } \\
\hline$<2,5$ & $1(3,3 \%)$ & \\
\hline $2,5-10$ & $21(70 \%)$ & \\
\hline $10-20$ & $2(6,67 \%)$ & \\
\hline$>20$ & $6(20 \%)$ & \\
\hline \multicolumn{3}{|c|}{ Grupo riesgo según biopsia } \\
\hline Alto & $11(36,7 \%)$ & \\
\hline Intermedio & $9(30 \%)$ & \\
\hline Bajo & $10(33,3 \%)$ & \\
\hline \multicolumn{3}{|c|}{ Afectación perineural } \\
\hline Sí & $6(15,8 \%)$ & \\
\hline No & $23(76,67 \%)$ & \\
\hline Desconocido & 1 & \\
\hline \multicolumn{3}{|l|}{ Gleason } \\
\hline 6 & $10(26,3 \%)$ & \\
\hline 7 & $11(36,67 \%)$ & \\
\hline 8 & $5(13,2 \%)$ & \\
\hline 9 & $4(10,5 \%)$ & \\
\hline
\end{tabular}


Extracción de microARN y reacción en cadena de la polimerasa a tiempo real con transcriptasa inversa cuantitativa

Se toman muestras de sangre periférica de pacientes antes de la terapia y de controles en tubos que contienen EDTA. Para aislar la fracción de miARN usamos el kit Ribopure siguiendo el protocolo de aislamiento conservando ARN pequeños (Applied Biosystems, Foster City, CA, EE. UU.). El procedimiento fue realizado usando $0,5 \mathrm{ml}$ de sangre total. Estos estudios de expresión diferencial entre grupos de riesgo con $\mathrm{CaP}$ fueron llevados a cabo sobre unas membranas de oligonucleótidos de la casa Exiqon en placas de 96 pocillos que incluían 92 miARN humanos recogidos en la base de datos miRBASE 16.0 (http://www.mirbase.org/). La PCR a tiempo real fue realizada en el LightCycler ${ }^{\circledR} 480$ Instrument (Roche, Mannheim, Alemania).

La expresión relativa para cada miARN en estudio fue calculada mediante la herramienta Relative Expression Software Tool (REST) ${ }^{8}$.

\section{Diseño de la matriz de microARN}

Los genes estudiados en la membrana de oligonucleótidos quedan recogidos en el anexo 1 (material suplementario)

Las secuencias de ARN a analizar se descargaron de las bases de datos de Sanger mirbase $16^{9}$ (www.mirbase.org/). Las secuencias recogidas en el anexo 1 se estudiaron mediante los programas microRNA.org ${ }^{10}$ (www.microrna.org/), dbDEMC ${ }^{11}$ (http://159.226.118.44/dbDEMC/index.html.), smiRNAdb $^{12}$ (www.mirz.unibas.ch/cloningprofiles/), GeneHUB-GEPIS ${ }^{13} \quad$ (http://researchpublic.gene.com/Research/genentech/genehub-gepis/index.html) y $\operatorname{miRex}^{14}$ (http://miracle.igib.res.in/mirex/) para simular situaciones mediante el uso de datos de expresión que surgen de experimentos previos. El estudio in silico de los perfiles de expresión de miARN se realizó utilizando las bases de datos arriba mencionadas para obtener información de aquellos marcadores miARN con mínima expresión en sangre normal y máxima en diferentes tipos de muestras como tejido de adenocarcinoma prostático, líneas celulares LNCap y PC3 y biopsias de tumor de próstata obtenidas con aguja gruesa, simulando en cada caso nuestro estudio in vivo posterior realizado en sangre periférica de pacientes afectados de $\mathrm{CaP}$ de bajo, intermedio y alto riesgo frente a los casos sanos usados como controles.

\section{Métodos estadísticos}

El análisis estadístico fue realizado usando el paquete estadístico SPSS 19.0 para Windows (SPSS, Chicago, IL) y se consideró la significación estadística a nivel 0,05.

\section{Resultados}

\section{MicroARN circulantes en grupos de riesgo de cáncer de próstata y controles}

Para investigar los niveles de expresión diferencial de los miARN circulantes mediante la técnica de PCR a tiempo real comparamos los perfiles de expresión de miARN usando el programa REST entre los distintos grupos de riesgo de CaP y el grupo control de casos sanos. De esta manera obtuvimos un listado de miARN upregulated y downregulated para los grupos de riesgo alto, intermedio y bajo en relación con la biopsia prostática. Dentro del grupo de alto riesgo encontramos 68 miARN circulantes upregulated y 3 downregulated, dentro del de riesgo intermedio 12 upregulated y 32 downregulated $\mathrm{y}$, por último, el grupo de bajo riesgo solo nos ofreció 37 miARN downregulated. Destacar por orden de mayor a menor expresión relativa el hsamiR-337-3p, hsa-miR-330-3p, hsa-miR-339-3p, hsa-miR-124, hsa-miR-218, hsa-miR-128, hsamiR-10a, hsa-miR-199b-5p, hsa-miR-200b y hsa-miR-15b. La figura 1 y el anexo 1 (material 
suplementario) muestra los gráficos en relación con la expresión de estos marcadores en los 3 grupos de riesgo.
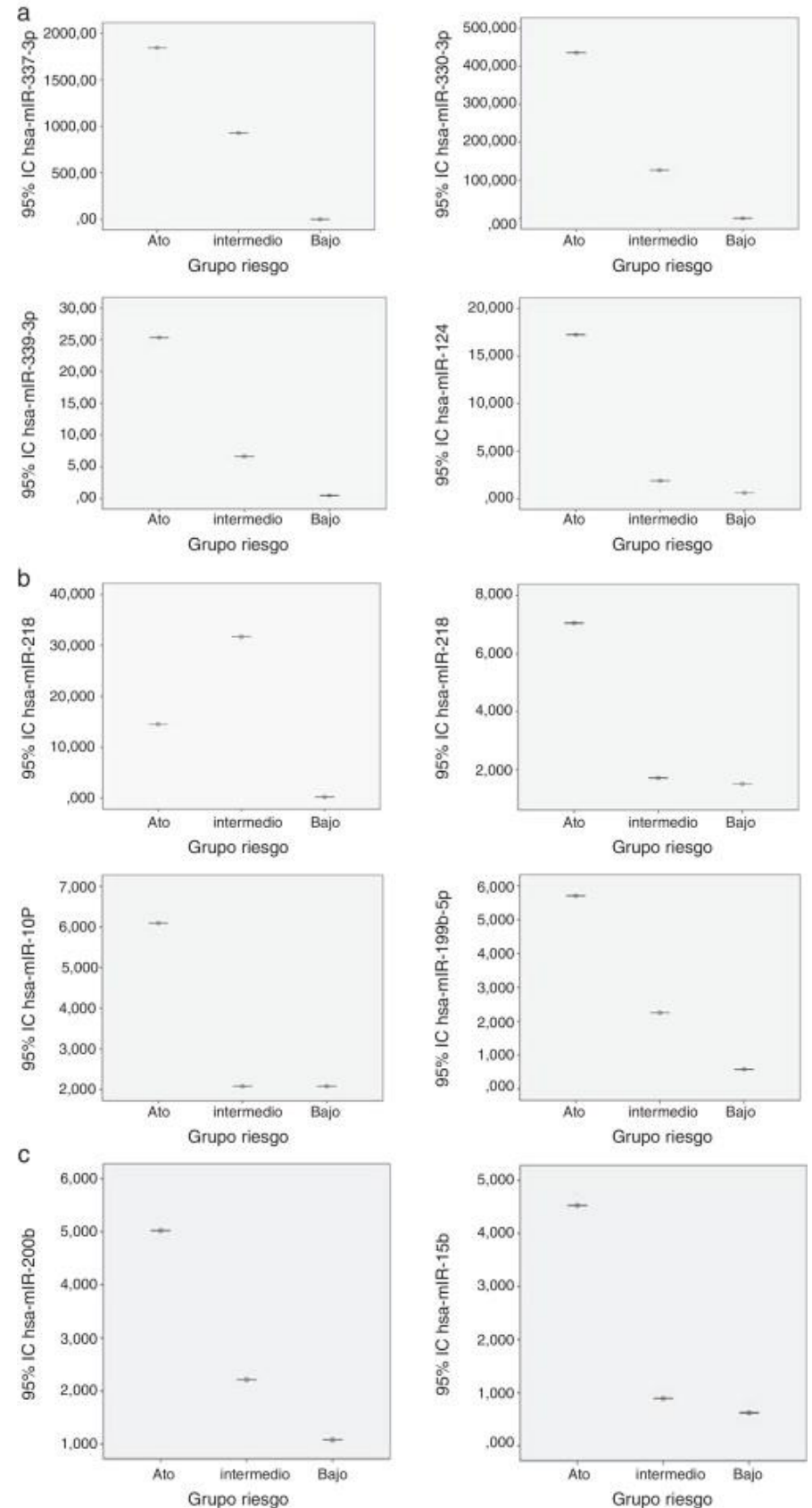

Figura 1. A. Medias de expresión de microARN candidatos por grupos de riesgo. La figura muestra la distribución de las medias para los marcadores miR337-3p, miR330-3p, miR339-3p y miR214 en relación con los 3 grupos de riesgo estudiados en la población de pacientes. B. Medias de expresión de microARN candidatos por grupos de riesgo. La figura muestra la distribución de las medias para los marcadores miR218, miR103, miR128 y miR199b-5p en relación con los 3 grupos de riesgo estudiados en la población de pacientes. C. Medias de expresión de microARN candidatos por grupos de riesgo. La figura muestra la distribución de las medias para los marcadores miR200b y miR15b en relación con los 3 grupos de riesgo estudiados en la población de pacientes. 
Usando el software de análisis genómico Multi Experiment Viewer 4.6.2 $(\mathrm{MeV})^{15}$, construimos un heatmap ( fig. 2) con 92 miARN expresados diferencialmente entre los 3 grupos de riesgo en estudio y la población sana. Se realizó una técnica de clasificación jerárquica de datos para realizar un estudio de cuántos patrones de expresión de miARN se obtenían y el resultado fueron 4 tipologías de expresión. La primera tipología comprendería a aquellos miARN que son menos expresados por la población en estudio, y así en orden creciente hasta llegar a la tipología 4 que reúne los miARN que son más expresados por la población estudiada.

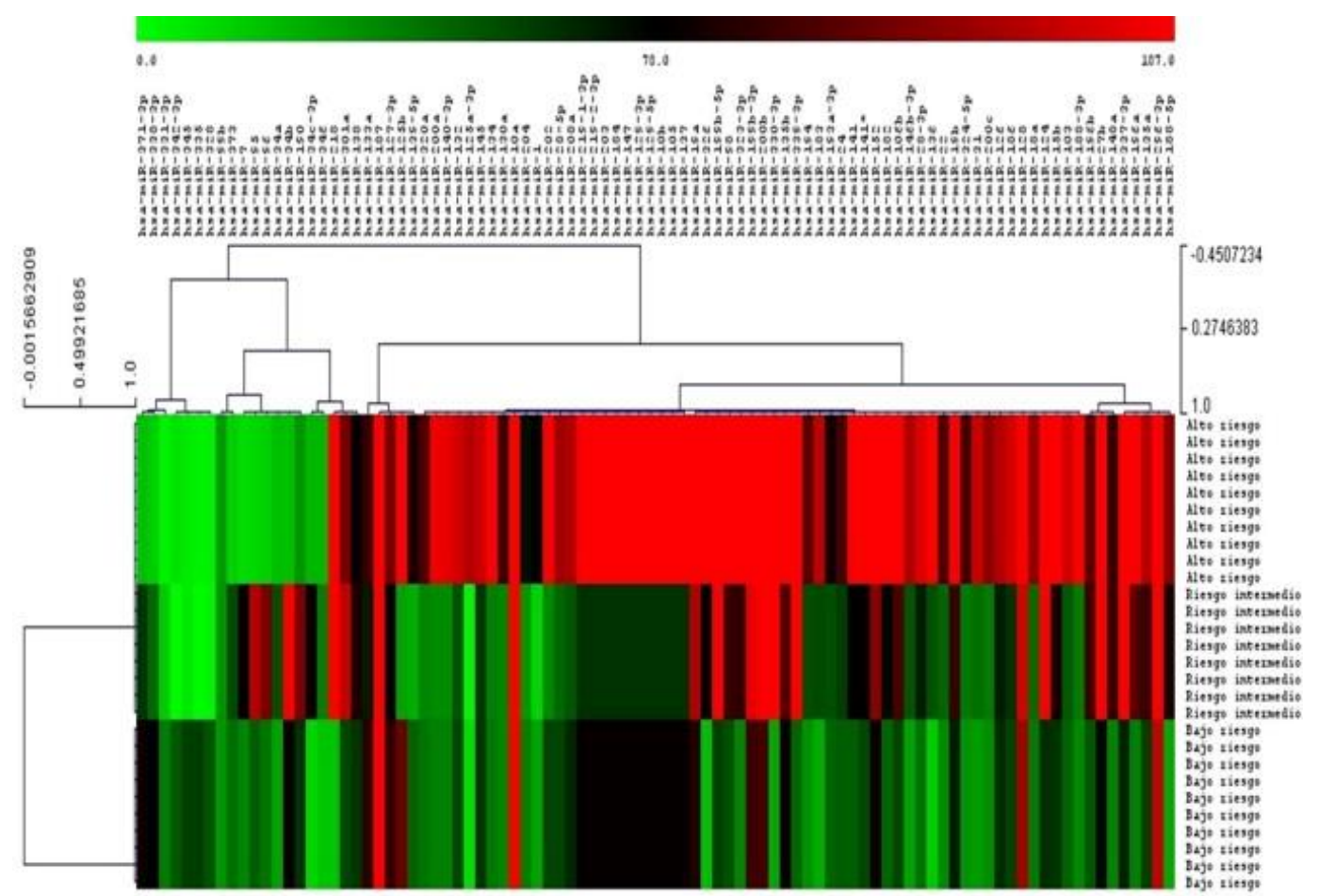

Figura 2. HeatMap. Se realizó una técnica de clasificación jerárquica de datos para realizar un estudio de cuántos patrones de expresión de microARN se obtenían. La figura muestra 4 tipologías de expresión y los microARN que las comprenden. La primera tipología comprendería aquellos microARN que son menos expresados por la población en estudio, y así en orden creciente hasta llegar a la tipología 4 que reúne los microARN que son más expresados por la población estudiada.

\section{Información diagnóstica}

La hipótesis de nuestro estudio es pronosticar los valores de una serie de variables nominales, entre ellas el grupo de riesgo en relación con la biopsia prostática, la escala de Gleason y la afectación perineural, prediciendo así qué pacientes serán incluidos en una u otra de las categorías de las variables dependientes a estudio. Para contrastar la hipótesis planteada hemos tomado en consideración los diferentes miARN estudiados que actuarán como indicadores. Para realizar predicciones acerca de las variables grupo de riesgo y escala de Gleason usamos la técnica de análisis multivariante regresión lineal $\left(\mathrm{R}^{2}\right.$ corregida $\left.=1, \mathrm{p}<0,01\right)$ en la cual los 92 miARN en estudio son combinados para realizar un correcto diagnóstico de la variable dependiente grupo de riesgo englobando los marcadores hsa-miR-188-5p, hsa-miR-187 y hsa-miR-196b.

Para la variable dependiente escala de Gleason la ecuación de regresión $\left(\mathrm{R}^{2}\right.$ corregida $=0,866$, $\mathrm{p}<0,01)$ engloba al marcador hsa-miR-135a. 
Por último la variable dicotómica afectación perineural fue estudiada mediante la técnica de regresión binaria. Solo el marcador hsa-miR-339-3p forma parte de la ecuación de regresión logística binaria $(\mathrm{p}=0,022)$. La clasificación por parte de este marcador en relación con la variable dependiente es correcta en el 76,2\% de los casos con no afectación perineural y en un 83,3\% para los casos que sí presentan afectación perineural. El marcador hsa-miR-339-3p aparece como un marcador de mal pronóstico, ya que el riesgo relativo $\operatorname{Exp}(B)=1,146$.

\section{Discusión}

Dentro de los tumores sólidos el $\mathrm{CaP}$ es una de las enfermedades oncológicas de mayor incidencia en países occidentales. $\mathrm{El} \mathrm{CaP}$ es actualmente reconocido como una enfermedad multifactorial. A pesar de que se reconoce un aporte ambiental al desarrollo del cáncer de próstata, se cree que la predisposición genética cumple un rol importante en el desarrollo de la enfermedad $^{16}$. Últimamente los trabajos de genómica están proporcionando el material que permitirá en un futuro cercano realizar una clasificación a nivel molecular, que permita distinguir los diferentes subtipos y establecer una mejor estratificación del $\mathrm{CaP}^{17}, 18,19$ and 20.

Recientemente se han reportado varios perfiles de expresión de miARN en tumores de próstata y todos coinciden en una extensa desregulación global de los mismos ${ }^{21}$. Además, fue demostrado que los patrones de expresión diferencial de los miARN en el cáncer de próstata pueden correlacionarse robustamente con la clínica, utilizándolos como indicadores de diagnóstico y pronóstico $^{22}$. Existen un gran número de miARN que han sido hallados expresados anormalmente en cáncer de próstata, asociados directamente al desarrollo del mismo. Muchos miARN (oncomirs) se encuentran sobreexpresados en el $\mathrm{CaP}$ regulando negativamente muchos genes supresores de tumor, conduciendo al crecimiento tumoral y a la metástasis. Por ejemplo, el hsa-mir-221 y hsamir-222 han sido reportados sobreexprados en $\mathrm{CaP}$, encontrándose directamente relacionados con la metástasis y el crecimiento tumoral a través de la represión del gen blanco p27kip1 $1^{23}$. Otro miARN que presenta función oncomir es el gen hsa-mir-21; este se encuentra sobreexpresado en el $\mathrm{CaP}$, participando en el crecimiento tumoral, así como en los procesos de invasión y metástasis. Por otro lado, el rol tumor supresivo de los miARN en el CaP se encuentra asociado con la habilidad de interferir con la migración celular y la invasión, así como mediar o promover la apoptosis celular. De hecho, la pérdida de miARN supresores de tumor es un mecanismo bastante común asociado al CaP. Por ejemplo, la pérdida del locus del hsa-mir-101 asociado a la sobreexpresión del gen EZH2 se encontró en un 37,5\% de muestras clínicas de cáncer de próstata localizado y en un $66,7 \%$ de muestras de cáncer de próstata metastásico. Asimismo, la sobreexpresión del hsa-mir-101 se encuentra asociada con niveles bajos de EZH2, así como con la supresión del crecimiento y de la invasividad en células de $\mathrm{CaP}^{24}$ and 25 . En nuestro trabajo hemos estudiado el perfil de expresión de 92 miARN circulantes en 30 enfermos con $\mathrm{CaP}$ y 10 controles sanos para poder conocer los perfiles de expresión de estos miARN según el grupo de riesgo en relación con la biopsia de próstata y poder asociar la expresión diferencial de estos marcadores con diferentes variables clínico-patológicas. Según nuestro conocimiento este es el primer estudio que analiza la expresión de miARN en sangre total de pacientes con CaP localizado y controles. El estudio estadístico de los resultados nos reveló 10 candidatos como mejores marcadores con expresión diferencial entre grupos de riesgo de CaP y controles sanos: hsa-miR-337-3p, hsa-miR330-3p, hsa-miR-339-3p, hsa-miR-124, hsa-miR-218, hsa-miR-128, hsa-miR-10a, hsa-miR-199b5p, hsa-miR-200b y hsa-miR-15b.

Los miARN poseen las propiedades ideales de un biomarcador. En primer lugar, su expresión se haya frecuentemente desregulada en la mayoría de las enfermedades humanas. En segundo lugar, numerosos trabajos muestran que los miARN pueden ser utilizados para la estratificación, seguimiento, pronóstico e incluso terapéutica del cáncer ${ }^{26}$. En tercer lugar, desde hace poco se sabe que los miARN circulan en el plasma, suero y orina ${ }^{27}$. Esto permitiría monitorizar sus niveles en sangre, de manera poco invasiva para el paciente y con bajos costes para el sistema de salud. En cuarto lugar, los niveles de los mismos en sangre se mantienen estables por periodos de tiempo considerables. En quinto lugar, la cuantificación de miARN es un método estandarizado y de fácil implementación en el laboratorio de biología molecular. Finalmente, muy recientemente comenzaron a publicarse estudios que demuestran robustas correlaciones entre los niveles de 
miARN en sangre con la clínica del paciente ${ }^{7 \text { and } 28}$ (estratificación, pronóstico, respuesta terapéutica) para diversas enfermedades, evidenciando así el importante valor clínico de los miARN circulantes.

En nuestro estudio el análisis de asociación con la clínica del paciente nos mostró que en conjunto el estudio de hsa-miR-188-5p, hsa-miR-187 y hsa-miR-196b serían buenos predictores para conocer mediante una determinación sanguínea el grupo de riesgo de un paciente afectado con CaP. Para predecir la escala Gleason el análisis estadístico de nuestros datos nos mostró que solo con determinar en sangre el hsa-miR-135a y someterlo a una ecuación de regresión lineal es suficiente para predecirlo. Por último, en el caso de la variable clínica afectación perineural encontramos que el conocimiento de la expresión relativa en sangre del marcador hsa-miR-339-3p sería suficiente para determinar si un paciente presenta o no dicha afectación, con el conocimiento además de que se trata de un marcador de riesgo, es decir, aumenta el riesgo de padecer afectación perineural por cada unidad que se incrementa este marcador.

Las limitaciones de este estudio vienen de la mano de un tamaño muestral pequeño, hecho que será complementado a medio plazo con un panel más amplio de casos reclutados, suponiendo que la medición masiva de los candidatos de este estudio determinará de una manera más certera sus indicaciones y rentabilidad diagnóstica y pronóstica.

Resaltar que cuando consideramos publicaciones relacionadas con el potencial diagnóstico y clínico del estudio de miARN en fluidos nos encontramos con una gran heterogeneidad de estudios y resultados al respecto. Diferencias en los métodos de extracción, cuantificación y detección de los miARN, el tipo (pre-miARN o formas maduras) y número de ellos evaluados, su procedencia y momento de la obtención (suero, plasma o células sanguíneas obtenidas antes o tras la cirugía), asî como las características clínico-patológicas asociadas a cada uno de los pacientes en el estudio son variables a tener en cuenta a la hora de intentar explicar esas causas de heterogeneidad.

Nuestros resultados confirman publicaciones previas acerca dea) posibilidad de detectar miARN en sangre total; b) los niveles de expresión de miARN dados perfilan grupos de riesgo en $\mathrm{CaP}$; y c) ciertos miARN englobados en el estudio destacan como prometedores marcadores con potencial diagnóstico.

\section{Financiación}

Este trabajo ha sido financiado exclusivamente mediante una Beca de la Fundación para la Investigación en Urología del año 2011.

\section{Conflicto de intereses}

Los autores declaran no tener ningún conflicto de intereses.

\section{Agradecimientos}

Los autores expresan su agradecimiento a los pacientes por haber donado sus muestras, a Eva Blanco por el trabajo realizado y a los Servicios de Urología y Oncología Médica del CHUAC-XXIAC.

\section{Bibliografía}

1. B.P. Lewis, C.B. Burge, D.P. Bartel. Conserved seed pairing, often flanked by adenosines, indicates that thousands of human genes are microRNA targets. Cell, 120 (2005), pp. 5-20.

2. M. Valladares-Ayerbes, M. Reboredo, V. Medina-Villaamil, P. Iglesias-Díaz, M.J. Lorenzo-Patiño, M. Haz, et al.. Circulating miR-200c as a diagnostic and prognostic biomarker for gastric cancer. J Trans Med, 10 (2012), p. 186.

3. X. Chen, Y. Ba, X. Cai, Y. Yin, K. Wang, J. Guo, et al. Characterization of microRNAs in serum: A novel class of biomarkers for diagnosis of cancer and other diseases. Cell Res, 18 (2008), pp. 997-1006. 
4. P.S. Mitchell, R.K. Parkin, E.M. Kroh, B.R. Fritz, S.K. Wyman, E.L. Pogosova-Agadjanyan, et al. Circulating microRNAs as stable blood-based markers for cancer detection. Proc Natl Acad Sci USA, 105 (2008), pp. 10513-10518.

5. J.C. Brase, M. Johannes, T. Schlomm, M. Falth, A. Haese, T. Steuber, et al. Circulating miRNAs are correlated with tumor progression in prostate cancer. Int J Cancer, 128 (2011), pp. 608-616.

6. L.A. Selth, S. Townley, J.L. Gillis, A.M. Ochnik, K. Murti, R.J. Macfarlane, et al. Discovery of circulating microRNAs associated with human prostate cancer using a mouse model of disease. Int J Cancer, 131 (2012), pp. 652-661.

7. R.J. Bryant, T. Pawlowski, J.W. Catto, G. Marsden, R.L. Vessella, B. Rhees, et al. Changes in circulating microRNA levels associated with prostate cancer. Br J Cancer, 106 (2012), pp. 768-774.

8. M.W. Pfaffl, G.W. Horgan, L. Dempfle. Relative expression software tool (REST) for group-wise comparison and statistical analysis of relative expression results in real-time PCR. Nucleic Acids Res, 30 (2002), p. e36.

9. A. Kozomara, S. Griffiths-Jones. miRBase: Integrating microRNA annotation and deep-sequencing data. Nucleic Acids Res, 39 (Database issue) (2011), pp. D152-D157.

10. D. Betel, M. Wilson, A. Gabow, D.S. Marks, C. Sander. The microRNA.org resource: Targets and expression. Nucleic Acids Res, 36 (Database issue) (2008), pp. D149-D153.

11. Z. Yang, F. Ren, C. Liu, S. He, G. Sun, Q. Gao, et al. dbDEMC: A database of differentially expressed miRNAs in human cancers. BMC Genomics, 2 (11 Suppl 4) (2010), p. S5.

12. P. Landgraf, M. Rusu, R. Sheridan, A. Sewer, N. Iovino, A. Aravin, et al. A mammalian microRNA expression atlas based on small RNA library sequencing. Cell, 129 (2007), pp. 1401-1414.

13. Y. Zhang, S.M. Luoh, L.S. Hon, R. Baertsch, W.I. Wood, Z. Zhang. GeneHub-GEPIS: Digital expression profiling for normal and cancer tissues based on an integrated gene database. Nucleic Acids Res, 35 (Web Server issue) (2007), pp. W152-W158.

14. R. Bargaje, M. Hariharan, V. Scaria, B. Pillai. Consensus miRNA expression profiles derived from interplatform normalization of microarray data. RNA, 16 (2010), pp. 16-25.

15. A.I. Saeed, V. Sharov, J. White, J. Li, W. Liang, N. Bhagabati, et al. TM4: A free, open-source system for microarray data management and analysis. Biotecniques, 34 (2003), p. 374.

16. M. Kral, V. Rosinska, V. Student, M. Grepl, M. Hrabec, J. Bouchal. Genetic determinants of prostate cancer: A review. Biomed Pap Med Fac Univ Palacky Olomouc Czech Repub, 155 (2011), pp. 3-9.

17. S.A. Tomlins, D.R. Rhodes, J. Yu, S. Varambally, R. Mehra, S. Perner, et al. The role of SPINK1 in ETS rearrangement-negative prostate cancers. Cancer Cell., 13 (2008), pp. 519-528.

18. N. Palanisamy, B. Ateeq, S. Kalyana-Sundaram, D. Pflueger, K. Ramnarayanan, S. Shankar, et al. Rearrangements of the RAF kinase pathway in prostate cancer, gastric cancer and melanoma. Nat Med, 16 (2010), pp. 793-798.

19. B.S. Taylor, N. Schultz, H. Hieronymus, A. Gopalan, Y. Xiao, B.S. Carver, et al. Integrative genomic profiling of human prostate cancer. Cancer Cell, 18 (2010), pp. 11-22.

20. M.M. Shen, C. Abate-Shen. Molecular genetics of prostate cancer: New prospects for old challenges. Genes Dev, 24 (2010), pp. 1967-2000.

21. P. Gandellini, M. Folini, N. Zaffaroni. Towards the definition of prostate cancer-related microRNAs: Where are we now?. Trends Mol Med, 15 (2009), pp. 381-390.

22. A. Schaefer, M. Jung, G. Kristiansen, M. Lein, M. Schrader, K. Miller, et al. Suitable reference genes for relative quantification of miRNA expression in prostate cancer. Exp Mol Med, 42 (2010), pp. 749-758.

23. T. Sun, Q. Wang, S. Balk, M. Brown, M. Lee, P. Kantoff. The role of microRNA 221 and microRNA-222 in androgen-independent prostate cancer cell lines. Cancer Res, 69 (2009), pp. 3356-3363.

24. S. Varambally, Q. Cao, R.S. Mani, S. Shankar, X. Wang, B. Ateeq, et al. Genomic loss of microRNA-101 leads to overexpression of histone methyltransferase EZH2 in cancer. Science, 322 (2008), pp. 1695 1699.

25. Y. Pang, C.Y. Young, H. Yuan. MicroRNAs and prostate cancer. Acta Biochim Biophys Sin (Shanghai), 42 (2010), pp. 363-369.

26. S.M. Metias, E. Lianidou, G.M. Yousef. MicroRNAs in clinical oncology: At the crossroads between promises and problems. J Clin Pathol, 62 (2009), pp. 771-776.

27. J.A. Weber, D.H. Baxter, S. Zhang, D.Y. Huang, K.H. Huang, M.J. Lee, et al. The microRNA spectrum in 12 body fluids. Clin Chem, 56 (2010), pp. 1733-1741.

28. Z.H. Chen, G.L. Zhang, H.R. Li, J.D. Luo, Z.X. Li, G.M. Chen, et al. A panel of five circulating MicroRNAs as potential biomarkers for prostate cancer. Prostate, 72 (2012), pp. 1443-1452. 